\title{
Relazioni fra produttività di un allevamento, composizione chimica e proprietà igienico- sanitarie del latte per la trasformazione in Parmigiano Reggiano
}

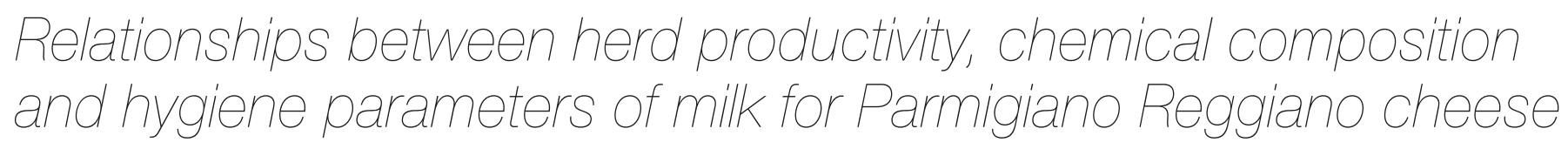

Dipartimento di Scienze Medico-Veterinarie, Università degli Studi di Parma, via del Taglio 10, 43126, Parma

*Corresponding author:

Piero Franceschi

Dipartimento di Scienze Medico-Veterinarie, Università degli Studi di Parma via del Taglio 10, 43126, Parma; piero.franceschi@unipr.it; tel: +39 0521032615
Ricevuto il 22 luglio 2019

Accettato il 5 settembre 2019

DOI: 10.36138/STLC.01.2019.01

\section{Riassunto}

L'obiettivo della ricerca è stato confrontare i principali parametri qualitativi del latte, utilizzati nel sistema pagamento a qualità, nell'ambito di stalle con vacche a differente produttività. La ricerca è stata condotta su 4'996 campioni di latte di singolo allevamento, raccolti da 150 aziende in un periodo di 10 anni. Sui campioni di latte di massa, di singolo allevamento, sono state effettuate le seguenti determinazioni: grasso e proteina, acidità titolabile, conta batterica totale, cellule somatiche, batteri coliformi, conta spore di clostridi. Le medie stimate sono state saggiate con ANOVA univariata. All'aumentare della dimensione dell'alleva- mento si osserva un aumento della produzione di latte e all'aumentare della produzione di latte si assiste ad una diminuzione progressiva del suo contenuto di grasso, della conta batterica totale e dell'inquinamento da batteri coliformi, del contenuto di cellule somatiche e dell'inquinamento da spore di clostridi.
Parole chiave:
- latte di massa
qualità del latte
composizione latte
parametri igienico sanitari
- Parmigiano Reggiano

\section{Abstract}

The aim of this research is to compare milk quality parameters among herd characterized by a different level of milk production. The research was carried out on 4,996 herd milk samples collected from 150 dairy herds during a period of 10 years. On each milk sample the following parameters were determined: fat and crude protein, titratable acidity, total bacterial count, somatic cells, coliforms bacteria and clostridia spores. Least mean values were obtained by ANOVA univariate. Milk production increased with herd size. Increased milk production was associated with a reduction of milk fat content total bacterial count, number of coliforms, somatic cells and number of $\mathrm{clo}$ stridia spores.

\section{Key words:}

Herd milk

Milk quality

Milk chemical composition

Hygienic-sanitary properties

— Parmigiano Reggiano cheese 


\section{INTRODUZIONE}

II Parmigiano Reggiano è un formaggio a pasta cotta, dura, a lunga stagionatura e a denominazione di origine protetta (DOP); come da disciplinare la produzione del latte e la trasformazione in formaggio avvengono esclusivamente nelle province di: Parma, Reggio Emilia, Modena, Bologna alla sinistra del fiume Reno e Mantova alla destra del fiume Po (www.parmigiano-reggiano. it). II Parmigiano Reggiano è ottenuto dalla lavorazione del latte della mungitura della sera (parzialmente scremato per affioramento naturale) miscelato con il latte intero della mungitura della mattina.

Nella produzione di Parmigiano Reggiano, come in quella di tutti i formaggi a latte crudo, la composizione chimica del latte e le sue caratteristiche microbiche hanno effetti significativi sulla resa casearia [1, 2] e sulla qualità del formaggio ottenuto [3]. Nella produzione del Parmigiano Reggiano la resa in formaggio è quindi direttamente correlata al contenuto di grasso e proteina del latte [4] ma risulta influenzata, anche, da parametri igienico-sanitari del latte, come ad esempio il contenuto di cellule somatiche $[2,5]$. Inoltre, sia un'elevata conta batterica totale che la presenza di batteri coliformi e spore di clostridi butirrici possono causare difetti strutturali, di colore e sensoriali nel formaggio [6].

La produzione di Parmigiano Reggiano, quindi, necessita di un latte con peculiari caratteristiche qualitative, indispensabili per conseguire un prodotto tipico e di pregio.

In generale, molti sono i fattori genetici [7], fisiologici ed ambientali $[2,8,9]$ che influenzando le vacche possono fortemente connotare la produttività per capo, e di conseguenza la redditività dell'allevamento con notevoli ripercussioni sulle caratteristiche degli stessi (dimensione della stalla e tipologia dei ricoveri, sistemi di alimentazione, tipologia degli impianti di mungitura e di stoccaggio del latte in allevamento, etc.). Tutti questi fattori, singolarmente presi o attraverso più o meno complesse interazioni, in virtù della loro complessità funzionale e delle notevoli e peculiari caratteristiche che li contraddistinguono, possono fortemente connotare la qualità del latte prodotto in azienda.

Allo scopo di migliorare le caratteristiche del latte destinato alla trasformazione in Parmigiano Reggiano, da parecchi anni è ormai consolidato un programma di pagamento differenziato in base alla qualità, che permette di remunerare il latte in rapporto a determinati requisiti chimici, fisico-chimici, microbiologici ed igienico-sanitari. L'obiettivo della ricerca è stato confrontare i principali parametri qualitativi del latte, utilizzati nel sistema pagamento a qualità, nell'ambito di stalle con vacche a differente produttività.

\section{MATERIALI E METODI}

La ricerca è stata condotta su 4'996 campioni di latte di singolo allevamento, provenienti da 150 aziende situate in provincia di Reggio Emilia, appartenenti al comprensorio di produzione del Parmigiano Reggiano. I prelievi sono stati effettuati nel corso di sopralluoghi mensili, nell'ambito del sistema pagamento latte a qualità, nel corso di dieci anni.

Sui campioni di latte di massa, di singolo allevamento, secondo quanto descritto da Franceschi et al (2012) [10] sono state effettuate le seguenti determinazioni: grasso e proteina, mediante letture nel medio infrarosso con apparecchio Milko-Scan della Foss (Foss Electric, DK-3400 Hillerød Denmark); acidità titolabile per titolazione di $50 \mathrm{~mL}$ di latte con soda $0,25 \mathrm{~N}$ secondo SoxhletHenkel; conta batterica totale con apparecchio BactoScan FC (Foss Electric); cellule somatiche mediante Fossomatic 5000 (Foss Electric); batteri coliformi mediante conta colonie su piastra dopo semina in terreno VRBA-agar e incubazione a $37^{\circ} \mathrm{C}$ per 24 ore [10]; conta spore di clostridi con il metodo MPN (most probable number) proposto da Weinzirl e modificato da Annibaldi [10]. Le medie stimate, dei dati sono state saggiate con ANOVA univariata, tramite software SPSS 18.0 impiegando un modello fattoriale completo con i seguenti fattori fissi: $\mathrm{kg}$ di latte per capo/lattazione (4 livelli; inferiore a 6.000 kg, da 6.000 a 7.999 kg, da $8^{\prime} 000$ a $10^{\prime} 000 \mathrm{~kg}$ ed oltre i $10000 \mathrm{~kg}$ di latte); consistenza dell'allevamento (5 livelli; inferiore a 30 capi allevati, da 30 a 60, da 61 a 100, da 101 a 200 e superiore a 200 capi); tipo di stabulazione (2 livelli; fissa e libera); stagione (4 livelli; inverno da gennaio a marzo, primavera da aprile a giugno, estate da luglio a settembre, autunno da ottobre a dicembre). La significatività delle differenze è stata saggiata mediante controllo LSD.

\section{RISULTATI E DISCUSSIONE}

Nelle tabelle I e II sono mostrate, rispettivamente, le correlazioni semplici tra le caratteristiche delle stalle e le principali caratteristiche del latte e le caratteristiche chimiche, fisico-chimiche e microbiologiche dei campioni di latte prelevati presso 150 stalle della provincia di Reggio Emilia.

La produzione di latte per capo/lattazione è risultata mediamente pari a $5714 \mathrm{~kg}$ per le vacche del gruppo $1,7 \cdot 225 \mathrm{~kg}$ per quelle del gruppo 2, 9'065 kg per quelle del gruppo 3 e 10'865 kg per quelle del gruppo 4 (dati non riportati in tabella).

Le stalle caratterizzate da più elevate produzioni di latte per capo sono risultate quelle con maggiore numero di vacche allevate $(r=0,257 ; P \leq 0,001)$. La consistenza media degli allevamenti, in- 


\section{Tabella I - Correlazioni semplici tra produzione di latte, consistenza degli allevamenti e i principali parametri chimici ed igienico-sanitari del latte.}

Table I - Pearson product moment between herd milk production level, herd size, milk chemical composition and hygiene parameter.

\begin{tabular}{|c|c|c|c|c|c|c|c|c|}
\hline & Consistenza $^{1}$ & $\begin{array}{c}\text { Conta } \\
\text { batterica }\end{array}$ & $\begin{array}{c}\text { Cellule } \\
\text { somatiche }\end{array}$ & $\begin{array}{l}\text { Batteri } \\
\text { coliformi }\end{array}$ & $\begin{array}{l}\text { Spore } \\
\text { clostridi }\end{array}$ & Grasso & Proteina & $\begin{array}{c}\text { Acidità } \\
\text { titolabile }\end{array}$ \\
\hline \multirow[t]{2}{*}{ kg latte ${ }^{2}$} & 0,257 & $-0,219$ & $-0,306$ & $-0,161$ & $-0,028$ & $-0,353$ & 0,036 & 0,139 \\
\hline & $\star \star \star ~$ & $\star \star \star \star ~$ & 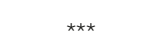 & $\star \star \star \star$ & * & $\star \star \star \star$ & * & $\star \star \star \star ~$ \\
\hline \multirow[t]{2}{*}{ Consistenza $^{1}$} & & $-0,130$ & 0,091 & $-0,077$ & 0,233 & $-0,209$ & 0,028 & 0,044 \\
\hline & & $\star \star \star \star ~$ & $\star \star \star \star ~$ & $\star \star \star$ & $\star \star \star \star ~$ & $\star \star \star \star ~$ & * & $\star \star$ \\
\hline
\end{tabular}

NS: $P>0,05 ;{ }^{*} \mathrm{P} \leq 0,05 ;{ }^{* \star} \mathrm{P} \leq 0,01 ;{ }^{* \star} \mathrm{P} \leq 0,001$.

${ }^{1}$ Numero vacche allevate

${ }^{2}$ Produzione latte (kg/vacca/lattazione)

fatti, è risultata essere minima nel gruppo 1 (32 capi allevati) e massima nel gruppo 4 (118 capi allevati) ed intermedia nei gruppi 2 (69 capi allevati) e 3 (88 capi allevati).

All'aumentare della produzione di latte per capo/lattazione è stata registrata una diminuzione del contenuto di grasso del latte $(r=$ $-0,353 ; \mathrm{P} \leq 0,001)$, che varia da un massimo di $3,73 \mathrm{~g} / 100 \mathrm{~g}$ nelle stalle del gruppo 1 ad un minimo di $3,25 \mathrm{~g} / 100 \mathrm{~g}$ nelle stalle del gruppo 4, con valori intermedi nelle stalle dei gruppi $2(3,48 \mathrm{~g} / 100 \mathrm{~g})$ e 3 (3,37 g/100g). Il contenuto medio di grasso, pari a 3,48, 3,37 e $3,25 \mathrm{~g} / 100 \mathrm{~g}$, osservato nel latte dei gruppi 2,3 e 4, rispettivamente, è da ritenersi basso se paragonato a quanto riportato da Summer et al. (2014) [8], che in uno studio durato 3 anni sul latte destinato alla trasformazione in formaggio Parmigiano Reggiano, individua un valore medio di 3,61 g/100g per il latte prodotto nelle stalle a stabulazione fissa e di 3,82 g/100g per quello prodotto nelle stalle a stabulazione libera. Questa elevata variabilità del contenuto di grasso del latte va ricercata nelle differenze nelle strategie di alimentazione. In effetti, nella maggior parte delle mandrie, con più di 50 vacche in allattamento, gli animali vengono alimentati attraverso la tecnica unifeed, mentre, in mandrie più piccole, i foraggi e i concentrati sono distribuiti separatamente (alimentazione tradizionale). La tecnica unifeed garantisce un migliore utilizzo dei nutrienti, consentendo un migliore sfruttamento del potenziale genetico delle bovine [11].

Il contenuto di proteina, invece, ha manifestato una debole correlazione positiva con la produzione di latte per capo/lattazione $(r=$ $0,036$; $P \leq 0,05)$, ma il suo andamento tra i gruppi è risultato altale- nante; varia, infatti, da un massimo di $3,37 \mathrm{~g} / 100 \mathrm{~g}$ nel latte delle stalle del gruppo 1 ad un minimo di 3,28 $\mathrm{g} / 100 \mathrm{~g}$ nelle stalle del gruppo 3 per risalire ad un valore intermedio $3,30 \mathrm{~g} / 100 \mathrm{~g}$ nelle stalle del gruppo 4. Inoltre, benché significativa, la differenza del contenuto di proteina del latte nei diversi gruppi è da considerarsi di piccola entità.

I valori di conta batterica totale, cellule somatiche, batteri coliformi e spore di clostridi butirrici hanno mostrato differenze statisticamente significative tra il latte prodotto nelle stalle dei diversi gruppi. All'aumentare della produzione di latte per capo/lattazione si assiste ad una diminuzione della conta batterica totale $(r=-0,219$; $P \leq 0,001)$, del contenuto delle cellule somatiche $(r=-0,306$; $P \leq 0,001)$, dei batteri coliformi $(r=-0,161 ; P \leq 0,001)$ e delle spore di clostridi $(r=-0,028 ; P \leq 0,05)$.

Conta batterica totale e batteri coliformi risultano entrambi maggiori nel latte delle stalle dei gruppi 1 e 2 (110 e 88 000UFC/mL e 2 '225 e 1'662 UFC/mL rispettivamente) nei confronti di quello delle stalle dei gruppi 3 e 4 (54 e 51 000UFC/mL e 1 '044 e 687 UFC/mL rispettivamente) tutti per $\mathrm{P} \leq 0,001$. II latte prodotto nelle stalle con maggiore produzione di latte per capo/lattazione è da ritenersi quindi igienicamente meno inquinato rispetto a quello prodotto nelle stalle con minore produzione di latte per capo/lattazione.

Il contenuto delle cellule somatiche, invece, varia da un massimo di 394 000unità/mL nelle stalle del gruppo 1 ad un minimo di 199 000unità/mL nel latte delle stalle del gruppo 4 , con valori intermedi nel latte delle stalle stalle dei gruppi 2 (367 000unità/mL) e 3 (270 


\section{Tabella II - Caratteristiche chimiche e parametri igienico-sanitari di 4:996 campioni di latte della mungitura della mattina di stalle con diversa produzione media annua di latte per capo (Medie stimate \pm errore standard).}

Table II - milk chemical composition and hygiene parameters of 4,996 milk samples collected by herd with different production level (least square mane \pm standard error)

\begin{tabular}{|c|c|c|c|c|c|c|c|c|c|c|c|c|c|c|c|c|c|c|}
\hline & & \multicolumn{17}{|c|}{ Classi produzione di latte per capo } \\
\hline & & \multicolumn{4}{|c|}{$\begin{array}{l}\text { Gruppo } 1 \\
<6000 \mathrm{~kg} \\
\left(\mathrm{n}^{1}=460\right)\end{array}$} & \multicolumn{4}{|c|}{$\begin{array}{c}\text { Gruppo } 2 \\
6000 \div 7999 \mathrm{~kg} \\
\left(\mathrm{n}^{1}=1897\right)\end{array}$} & \multicolumn{4}{|c|}{$\begin{array}{c}\text { Gruppo } 3 \\
8000 \div 10000 \mathrm{~kg} \\
\left(\mathrm{n}^{1}=1864\right)\end{array}$} & \multicolumn{4}{|c|}{$\begin{array}{c}\text { Gruppo } 4 \\
>10000 \mathrm{~kg} \\
\left(n^{1}=775\right)\end{array}$} & $P^{3}$ \\
\hline Numero vacche & capi & 32 & \pm & 1,5 & a & 69 & \pm & 1 & $\mathrm{~b}$ & 88 & \pm & 0,7 & C & 118 & \pm & 1,1 & $d$ & 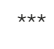 \\
\hline Grasso & $g / 100 g$ & 3,73 & \pm & 0,02 & d & 3,48 & \pm & 0,02 & C & 3,37 & \pm & 0,01 & $\mathrm{~b}$ & 3,25 & \pm & 0,02 & a & $\star \star \star ~$ \\
\hline Acidità titolabile & ${ }^{\circ} \mathrm{SH} / 50 \mathrm{~mL}$ & 3,26 & \pm & 0,01 & & 3,21 & \pm & 0,01 & & 3,24 & \pm & 0,01 & & 3,24 & \pm & 0,01 & & NS \\
\hline Conta batterica & 000/mL & 110 & \pm & 12 & $b$ & 83 & \pm & 8 & $\mathrm{~b}$ & 54 & \pm & 6 & $a$ & 51 & \pm & 9 & $\mathrm{a}$ & $\star \star \star ~$ \\
\hline Cellule somatiche & 000/mL & 394 & \pm & 11 & $d$ & 367 & \pm & 7 & c & 270 & \pm & 5 & $\mathrm{~b}$ & 199 & \pm & 8 & a & $\star \star \star *$ \\
\hline Batteri coliformi & UFC/mL & 2225 & \pm & 344 & $b$ & 1662 & \pm & 227 & $\mathrm{~b}$ & 1044 & \pm & 164 & $\mathrm{a}$ & 687 & \pm & 261 & a & 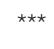 \\
\hline Spore clostridi & Spore/L & 124 & \pm & 8 & $\mathrm{C}$ & 102 & \pm & 6 & $\mathrm{~b}$ & 108 & \pm & 4 & $\mathrm{~b}$ & 74 & \pm & 6 & $\mathrm{a}$ & $\star \star \star$ \\
\hline
\end{tabular}

${ }^{1}$ Numero di campioni di latte di allevamento relativi alla mungitura della mattina

2 Errore standard

${ }^{3}$ Significatività statistica differenza: $a, b, c, d$ differenti per $P \leq 0,05 ;{ }^{* \star *} \mathrm{P} \leq 0,001$

000unità $/ \mathrm{mL}$ ) con $\mathrm{P} \leq 0,001$. I contenuti di cellule somatiche del latte dei gruppi 1 e 2 sono da ritenersi nella norma se paragonati a quanto riportato da Summer et al (2014) [8] e da Franceschi et al (2012) [9], che individuano un valore medio di 309000 unità $/ \mathrm{mL}$ per latte, prodotto in pianura e destinato alla trasformazione in formaggio Parmigiano Reggiano.

La tendenza per un conteggio delle cellule somatiche più elevato nei gruppi 1 e 2 appare in contrasto con i rapporti di Sadeghi-Sefidmazgi e Rayatdoost-Baghal (2014) [12] che hanno riscontrato un conteggio delle cellule somatiche del latte più basso in allevamenti meno produttivi (lattazione <10000 kg / 305 giorni). Questa differen- za è probabilmente dovuta alle diverse condizioni ambientali in cui sono stati condotti i due studi

Infine, l'inquinamento da spore di clostridi varia da un massimo di 124 spore/L nel latte delle stalle del gruppo 1 ad un minimo di 74 spore/L nel latte delle stalle del gruppo 4 , con valori intermedi nel latte delle stalle dei gruppi 2 (102 spore/L) e 3 (108 spore/L) sempre con $\mathrm{P} \leq 0,001$. I contenuti di spore di clostridi del latte dei gruppi 1,2 e 3 sono da ritenersi elevati, paragonati a quanto riportato da Summer et al 2014 [8] per il latte destinato alla trasformazione in formaggio Parmigiano Reggiano, che individua il valore di 100 spore/L come soglia per definire un latte inquinato da spore di clostridi. 


\section{CONCLUSIONI}

All'aumentare della dimensione dell'allevamento si osserva un aumento della produzione di latte $(r=0,257 ; P \leq 0,001)$. All'aumentare della produzione di latte per capo/lattazione si osserva una diminuzione del suo contenuto di grasso $(r=-0,353 ; P \leq 0,001)$; questo rilievo trova conferma nella correlazione semplice di segno negativo che vi è tra il numero delle vacche allevate e contenuto di grasso nel latte $(r=-0,209 ; P \leq 0,001)$.

Inoltre, sempre all'aumentare della produzione di latte per capo/lattazione si osserva anche un miglioramento generale dello stato igienico-sanitario del latte; infatti si assiste ad una diminuzione progressiva della conta batterica totale e dell'inquinamento da batteri coliformi, del contenuto delle cellule somatiche e dell'inquinamento da spore di clostridi.

\section{CONFLITTO DI INTERESSE}

Gli autori dichiarano di non avere conflitti di interesse con quanto riportato in questo articolo.

\section{BIBLIOGRAFIA}

1. Franceschi P, Malacarne M, Formaggioni P, Stocco G, Cipolat-Gotet C, Summer A (2019) Effect of season and cheese-factory on cheese-making efficiency in Parmigiano Reggiano cheese manufacture. Foods 8(8): 315-323.

2. Summer A, Franceschi P, Formaggioni $P$, Malacarne $M(2015)$ Influence of milk somatic cell content on Parmigiano-Reggiano cheese yield. J Dairy Res 82:222-227. 3. Vianna, PCB, Mazal G, Santos, MV, Bolini, HMA, Gigante, ML (2008) Microbial and sensory changes throughout the ripening of Prato cheese made from milk with different levels of somatic cells. J Dairy Sci 91:1743-1750.

4. Formaggioni P, Summer A, Malacarne M, Franceschi P, Mucchetti G (2015) Italian and Italian-style hard cooked cheeses: predictive formulas for Parmigiano-Reggiano $24 \mathrm{~h}$ cheese yield. Int Dairy J 51:52-58.

5. Le Maréchal C, Thiéry R, Vautor E, Le Loir Y (2011) Mastitis impact on technological properties of milk and quality of milk products - a review. Dairy Sci and Technol 91:247-282.

6. Beresford TP, Fitzsimons NA, Brennan, NL, Cogan TM (2011) Recent advances in cheese microbiology. Int Dairy J 11:59-274.

7. Heck JML, Schennink A, van Valenberg HJF, Bovenhuis H, Visker MHPW, van Arendonk JAM, et al. (2009) Effects of milk protein variants on the protein composition of bovine milk. J Dairy Sci 92:1192-202.
8. Summer A, Franceschi P, Formaggioni P, Malacarne M (2014) Characteristics of raw milk produced by free-stall or tie-stall cattle herds in the Parmigiano-Reggiano cheese production area. Dairy Sci Tech 94:581-590.

9. Franceschi P, Malacarne M, Nordera B, Vergnani M, Ghizzoni PG, Formaggioni P, et al. (2012) Effect of pedoclimatic conditions on physico-chemical characteristics, hygienic-sanitary quality and coagulation properties of milk for Parmigiano-Reggiano cheese. Milchwiss 67:166-169.

10. Franceschi P, Nordera B, Malacarne M, Sun W, Vergnani M, Ghizzoni PG, Formaggioni P, et al. (2012) Effetti della consistenza degli allevamenti su qualità chimica e igienico-sanitaria del latte destinato alla trasformazione in Parmigiano-Reggiano. Sci Tecn Latt-Cas 63:195-199.

11. Comino L, Righi F, Coppa M, Quarantelli A, Tabacco E, Borreani G (2015) Relationships among early lactation milk fat depression, cattle productivity and fatty acid composition on intensive dairy farms in northern Italy. It J Anim Sci 14:350-361.

12. Sadeghi-Sefidmazgi A, Rayatdoost-Baghal F (2014) Effects of herd management practices on somatic cell counts in an arid climate. Rev. Bra zoot 43: 499-504. 